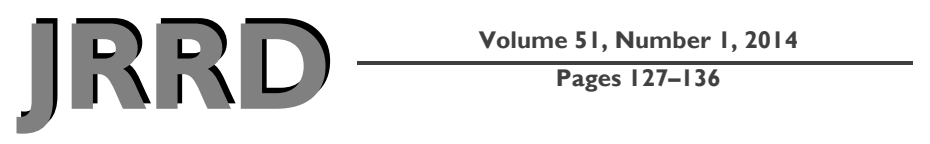

\title{
Comparison of mental health between individuals with spinal cord injury and able-bodied controls in Neiva, Colombia
}

\author{
Leia A. Harper, BS; ${ }^{1}$ Jennifer A. Coleman, MA; ${ }^{1}$ Paul B. Perrin, PhD; ${ }^{1}$ Silvia Leonor Olivera, MS; ${ }^{2}$ Jose Libardo \\ Perdomo, BS; ${ }^{3}$ Jose Anselmo Arango, BS; ${ }^{3}$ Juan Carlos Arango-Lasprilla, PhD $^{4^{*}}$ \\ ${ }^{1}$ Department of Psychology, Virginia Commonwealth University, Richmond, VA; ${ }^{2}$ Department of Psychology, Surcolombiana \\ University, Neiva, Colombia; ${ }^{3}$ Department of Psychology, Universidad Cooperativa de Colombia, Neiva, Colombia; \\ ${ }^{4}$ Department of Psychology, Ikerbasque, Basque Foundation for Science, University of Deusto, Bilbao, Spain
}

\begin{abstract}
Although research has investigated the mental health of individuals with spinal cord injury (SCI), an overwhelming majority of this research has been conducted in the United States, Western Europe, and other developed countries. The purpose of this study was to compare the mental health of individuals with SCI with able-bodied controls in Neiva, Colombia, South America. Subjects included 40 Colombians with SCI and 42 age- and sex-matched controls $(N=82)$. The groups did not differ based on age, sex, years of education, or socioeconomic status. However, controls were twice as likely to be married. Four measures assessed mental health, including satisfaction with life (Satisfaction with Life Scale), depressive symptoms (Patient Health Questionnaire-9), self-esteem (Rosenberg Self-Esteem Scale), and anxiety (State-Trait Anxiety Inventory). In comparison with ablebodied controls, individuals with SCI reported significantly lower mental health on both depressive symptoms and satisfaction with life. These effect sizes were medium and large, respectively. The groups did not differ significantly on measures of self-esteem or anxiety. Mental health of individuals with SCI should be considered a central part of SCI rehabilitation interventions, particularly in Latin America.
\end{abstract}

Key words: anxiety, Colombia, depression, Latin America, mental health, persons with disabilities, rehabilitation, satisfaction with life, self-esteem, spinal cord injury.

\section{INTRODUCTION}

Spinal cord injury (SCI) can be a life-altering event with a tremendous effect on the injured individual in multiple life domains. SCI is a chronic disorder that often begins with a traumatic injury to the spine and ends with damage to the spinal cord or nerves [1]. Globally, the average age of injury is $33 \mathrm{yr}$ [2], and due to its sudden occurrence, individuals are often unprepared to cope with the emotional, financial, and physical consequences of the injury and the subsequent disabilities. Motor vehicle collisions are the leading cause of SCI in developed countries like the United States, whereas in developing countries like Colombia, violence and falls from heights are more frequent causes [3].

\footnotetext{
Abbreviations: $\mathrm{ANOVA}=$ analysis of variance, $\mathrm{IRB}=$ institutional review board, MANOVA = multivariate analysis of variance, PHQ-9 = Patient Health Questionnaire-9, QoL = quality of life, RSES $=$ Rosenberg Self-Esteem Scale, SCI = spinal cord injury, SD = standard deviation, STAI = State-Trait Anxiety Inventory, SWLS = Satisfaction with Life Scale.

*Address all correspondence to Juan Carlos ArangoLasprilla, PhD; Department of Psychology, Ikerbasque, Basque Foundation for Science, University of Deusto, Bilbao, Spain; 804-859-4329. Email: jcarango@deusto.es http://dx.doi.org/10.1682/JRRD.2013.04.0086
} 
While the prognosis and life expectancy of individuals with SCI continue to improve, the effects are especially challenging for individuals in less-developed countries because outpatient rehabilitation hospitals, assistive technology, and other vocational rehabilitation services are extremely limited. Individuals with SCI generally have complicating medical conditions subsequent to the SCI that can permanently alter their lives. Medical complications may include bladder cancer, gallstones, renal stones, spasticity, problems with sexual functioning [4], recurrent pain, urinary tract infections, and ventilator dependence [1]. These complications may not only introduce a significant financial burden but can also complicate social and community reintegration [5].

In part because of this plethora of common medical issues, individuals with SCI may experience high levels of emotional distress related to their loss of independence and autonomy [6], the disruption in vocational advancement [7], deficits in physical functioning, and the resulting effect on social and intimate relationships [8]. Perhaps as a result, individuals with SCI have an increased risk of psychological and neurobehavioral problems such as anxiety, depression, and substance abuse [5,9]. Suicide rates for individuals with SCI in the United States are between two to six times higher than in the general population [10]. The prevalence of depression in individuals with SCI has been found to range from 8 to 60 percent, has been linked to lower satisfaction with life, and has been found to persist over time for many individuals [1113]. However, other researchers argue that depression develops only in a minority of individuals with SCI and changes dramatically across time since injury [14].

Directly related, pain and fatigue have been found to affect the rehabilitation process for individuals with SCI [15]. The prevalence of fatigue in individuals with longterm SCI is approximately 25 percent, and fatigue can result in higher depression and lower self-efficacy [1617]. Systematic reviews have indicated that negative affect is significantly associated with lower quality of life (QoL) and higher posttraumatic stress [18]. Psychiatric conditions such as these create barriers to rehabilitation and recovery, resulting in diminished QoL, increased risk for pain, and secondary medical complications [19].

Systematic reviews of mental health problems in individuals with SCI suggest that major depression, anxiety disorders, posttraumatic stress disorder, and substance abuse are extremely common [20]. Depression has been one of the more widely studied mental health concerns for individuals with SCI [12] and has been found to be significantly different than matched noninjured comparison groups [21]. Depressive symptoms have also been linked to other medical complications such as decubitus ulcers and urinary tract infections [22], as well as increased hospital stays, increased mortality, increased morbidity, and reduced functional improvements in rehabilitation [23].

Fewer studies have examined anxiety, satisfaction with life, and self-esteem in individuals with SCI. Research examining clinically significant anxiety in individuals with SCI shows that prevalence rates range from 13 to 40 percent and are significantly higher than in general comparison populations [12]. Research comparing anxiety levels in individuals with SCI with able-bodied comparison groups also show significant differences [21]. Rates tend to vary depending on time postinjury and individual differences. Lastly, research on self-esteem in individuals with SCI has shown mixed results [24].

Although a substantial amount of research has investigated the mental health of individuals with SCI, an overwhelming majority of this research has been conducted in the United States, Western Europe, and other developed countries. The incidence of traumatic SCI in Latin America is higher than in Western Europe and Australia, but lower than in the United States [25]. Few studies have examined whether racial, cultural, or global differences are present in the mental health of individuals with SCI [26]. However, research has found that white Americans with SCI report higher subjective well-being with regard to employment opportunities than African Americans, American Indians, and Latino Americans [27]. Latino Americans with SCI in the United States have been found to have higher rates of depression than white Americans and African Americans [26]. Research has also found that white individuals post-SCI have better employment outcomes than individuals from minority groups [26].

Given unique cultural, economic, and interpersonal differences, it is plausible that mental health could differ considerably among individuals with SCI in developing countries, such as Colombia. As such, the goal of the present study is to compare the mental health of individuals with SCI with a able-bodied comparison group in Neiva, Colombia, South America. We hypothesized that the mental health of individuals with SCI will be significantly lower than controls on measures of depressive symptoms, anxiety, self-esteem, and satisfaction with life. 


\section{METHODS}

\section{Participants}

Forty Colombian individuals with SCI were recruited from the Foundation for the Integral Development of People with Disabilities, a nonprofit organization that assists individuals with a variety of physical disabilities living in Neiva, Colombia. The age of the individuals with SCI was $34.8 \pm 11.0 \mathrm{yr}$ (mean \pm standard deviation [SD]), and 92.5 percent were male. More than half $(65.0 \%)$ were single, with an average of $10.0 \pm 4.6 \mathrm{yr}$ of education. Of the participants, 57.5 percent were at level 2 socioeconomic status as defined by the Colombian government (the Colombian government has defined 6 levels of socioeconomic status based on individual's income and location of residence, where level $1=$ the lowest resources and QoL and $6=$ highest). The time since injury was $142.6 \pm$ 104.0 mo, with 55.0 percent diagnosed with paraplegia and 35.0 percent of injuries resulting from violent events.

\section{Controls}

The control group was comprised of 42 individuals recruited through flyers and by general word of mouth. Their age was $35.6 \pm 11.0 \mathrm{yr}, 90.5$ percent of whom were male. Of the participants, 28.6 percent were single and 54.8 percent were at level 2 socioeconomic status, with an average of $9.9 \pm 4.2 \mathrm{yr}$ of education.

\section{Instruments}

\section{Satisfaction with Life Scale}

The Satisfaction with Life Scale (SWLS) is a selfreport questionnaire that assesses global life satisfaction [28]. The instrument consists of five items that the participant rates on a scale from 1 (strongly disagree) to 7 (strongly agree). Higher scores indicate a higher satisfaction with life. This study used the Spanish-language version of the SWLS, which has good psychometric properties [29-30].

\section{Patient Health Questionnaire-9}

The Patient Health Questionnaire-9 (PHQ-9) is selfreport measure that assesses depressive symptoms [31]. This scale consists of nine items that individuals rate using a 4-point Likert scale, where $0=$ not at all and $3=$ nearly every day. Scores are summed and range from 0 to 27 , with higher scores indicating higher levels of depressive symptoms. Respondents indicate how often they have been bothered by each of the items over the previous 2 wk. The Spanish-language version of the PHQ-9 was used in this study and has been found to be reliable and valid [32].

\section{Rosenberg Self-Esteem Scale}

The Rosenberg Self-Esteem Scale (RSES) is a selfreport questionnaire consisting of 10 items that assess selfesteem [33]. The respondents rate their overall sense of worth on a 4-point Likert scale, where $1=$ strongly disagree and $4=$ strongly agree [34], with total scores ranging from 10 to 40. Higher scores represent higher self-esteem, and a score of $<25$ suggests clinically significant low selfesteem [35]. The RSES is a reliable and valid measure that has shown good internal reliability with cross-cultural use in Latin America [34,36].

\section{State-Trait Anxiety Inventory}

The State-Trait Anxiety Inventory (STAI) is a 20item self-report measure of anxiety. The questionnaire includes two scales: the S-Anxiety scale that assesses anxiety as an emotional state and the T-Anxiety scale that assesses anxiety as a personality trait [37]. Each scale has response options ranging from 0 (almost never) to 3 (almost always). The total score, which was used in this study, ranges from 0 to 120 , and higher scores indicate higher levels of anxiety. The Spanish-language version has strong psychometric properties [38-39].

\section{Procedure}

Institutional review board (IRB) approval for this study was obtained from Surcolombiana University in Neiva, Colombia, and all approved informed consent and data privacy procedures were followed. The Foundation for the Integral Development of People with Disabilities is the only community resource available for individuals with physical disabilities living in Neiva and serves as a meeting place, social work organization, advocacy group, counseling service, and social support network. Research staff reviewed records at the Foundation in order to identify persons who had sought services for SCI between January 2005 and December 2008. Inclusion criteria required participants to have been diagnosed with an SCI after the age of 18; be at least 6 mo postinjury; and have no psychiatric problems, neurological problems, or history of drug and/or alcohol abuse according to Diagnostic and Statistical Manual-4th edition substance abuse criteria [40]. 
Out of a possible 42 patients identified in the medical records review, 2 had a reported history of drug or alcohol abuse and were excluded. The other 40 patients were called at home and invited to participate after receiving information about the study. All 40 individuals with SCI agreed to participate, comprising the full convenience SCI subsample used in this study. Controls were recruited from the same community as individuals with SCI through flyers at restaurants, churches, and stores, as well as by word of mouth.

All interviews were conducted in participants' homes by one of two psychologists, who both had an SCI themselves. The psychologists were supervised directly by a university professor. Participants gave informed consent under the IRB-approved protocol and then orally provided sociodemographic information. They then orally completed the study measures, and interviews lasted between 30 and $45 \mathrm{~min}$.

\section{Statistical Analysis}

Demographics for patients and controls were compared using $t$-tests for continuous variables and chisquare tests for nominal variables (Table 1). Demographic variables with statistically significant differences between patients and controls were compared in their prediction of the mental health variables under scrutiny to determine whether they should be covaried. In order to control for family-wise error, a multivariate analysis of variance (MANOVA) was conducted with participant status (patient vs control) as the independent variable and the four mental health indices of satisfaction with life, depressive symptoms, self-esteem, and anxiety as the dependent variables. A power analysis with $n=82$ was conducted using G*Power version 3 (Heinrich-HeineUniversität Düsseldorf; Düsseldorf, Germany) and determined that the MANOVA could detect $\left(f^{2}=0.25\right)$ most medium and all large effect sizes, having an actual power $(1-\beta)$ of 0.95 . Follow-up one-way analyses of variance (ANOVAs) were conducted to compare individuals with SCI and able-bodied controls on each mental health variable. For reference, Table 2 presents a correlation matrix showing the bivariate relationships between all dependent variables in the study. The data were analyzed using the statistical package SPSS, version 20 (IBM Corporation; Armonk, New York).

\section{RESULTS}

Normality assumptions were checked and met for all variables except for controls' scores on the PHQ-9 depressive symptom inventory (skewness $=1.85$, kurtosis $=$ 4.15). Because this variable was only slightly skewed and the only variable that departed from a normal distribution, it was not transformed.

Individuals with SCI and able-bodied controls had similar mean ages, sex distribution, years of education, and socioeconomic status levels (Table 1). Marital status was recoded with married and cohabiting individuals coded as partnered, and all other individuals coded as not partnered. Able-bodied controls were significantly more likely to be partnered than individuals with SCI (Table 1). When this effect was examined further, individuals with SCI were more likely to be single $(65.0 \%)$ than controls

Table 1.

Participant demographics.

\begin{tabular}{|c|c|c|c|}
\hline Variable & $\begin{array}{c}\text { Individuals } \\
\text { with SCI } \\
(n=40)\end{array}$ & $\begin{array}{c}\text { Able-Bodied } \\
\text { Controls } \\
(n=42)\end{array}$ & $p$-Value \\
\hline$\overline{\text { Age, } \text { yr }(\text { mean } \pm \text { SD) }}$ & $34.8 \pm 11.0$ & $35.6 \pm 11.0$ & NS \\
\hline $\operatorname{Sex}(\%)$ & & & NS \\
\hline Male & 92.5 & 90.5 & \\
\hline Female & 7.5 & 9.5 & \\
\hline Marital Status ${ }^{*}(\%)$ & & & 0.01 \\
\hline Single & 65.0 & 28.6 & \\
\hline Married & 17.5 & 38.1 & \\
\hline Divorced & 0.0 & 2.4 & \\
\hline Separated & 0.0 & 2.4 & \\
\hline Widow & 0.0 & 2.4 & \\
\hline Cohabitating & 17.5 & 26.2 & \\
\hline Education, yr (mean $\pm \mathrm{SD}$ ) & $10.0 \pm 4.6$ & $9.9 \pm 4.2$ & NS \\
\hline Socioeconomic Status ${ }^{\dagger}(\%)$ & & & NS \\
\hline Level 1 & 22.5 & 23.8 & \\
\hline Level 2 & 57.5 & 54.8 & \\
\hline Level 3 & 10.0 & 11.9 & \\
\hline Level 4 & 10.0 & 9.5 & \\
\hline Level of Injury (\%) & & & - \\
\hline Tetraplegia & 45.0 & - & \\
\hline Paraplegia & 55.0 & - & \\
\hline Cause of Injury (\%) & & & - \\
\hline Violent & 35.0 & - & \\
\hline Nonviolent & 65.0 & - & \\
\hline Time Since Injury, mo (mean \pm SD) & $142.6 \pm 104.0$ & - & - \\
\hline \multicolumn{4}{|c|}{$\begin{array}{l}\text { *Marital status tested as partnered (yes/no). } \\
{ }^{\dagger} \text { Colombian government defines } 6 \text { levels of socioeconomic status based on } \\
\text { individual's income and location of residence, where level } 1=\text { lowest resources } \\
\text { and quality of life and } 6=\text { highest. } \\
\text { NS = nonsignificant }(p>0.05), \mathrm{SCI}=\text { spinal cord injury, SD }=\text { standard deviation. }\end{array}$} \\
\hline
\end{tabular}


Table 2.

Correlation matrix.

\begin{tabular}{|c|c|c|c|c|}
\hline Participants & $\begin{array}{c}\text { Satisfaction } \\
\text { with Life } \\
\text { (SWLS) }\end{array}$ & $\begin{array}{c}\text { Depressive } \\
\text { Symptoms } \\
\text { (PHQ-9) }\end{array}$ & $\begin{array}{l}\text { Self-Esteem } \\
\text { (RSES) }\end{array}$ & $\begin{array}{c}\text { Anxiety } \\
\text { (STAI) }\end{array}$ \\
\hline \multicolumn{5}{|l|}{ Individuals with SCI } \\
\hline Satisfaction with Life & - & & & \\
\hline Depressive Symptoms & $-0.359^{*}$ & - & & \\
\hline Self-Esteem & $0.270^{*}$ & $-0.591^{\dagger}$ & - & \\
\hline Anxiety & $-0.376^{\dagger}$ & $0.726^{\dagger}$ & $-0.737^{\dagger}$ & - \\
\hline \multicolumn{5}{|l|}{ Able-Bodied Controls } \\
\hline Satisfaction with Life & - & & & \\
\hline Depressive Symptoms & $-0.370^{\dagger}$ & - & & \\
\hline Self-Esteem & $0.417^{\dagger}$ & $-0.531^{\dagger}$ & - & \\
\hline Anxiety & $-0.428^{\dagger}$ & $0.770^{\dagger}$ & $-0.522^{\dagger}$ & - \\
\hline \multicolumn{5}{|c|}{$\begin{array}{l}{ }^{*} \text { Significant at } 0.05 \text { level. } \\
{ }^{\dagger} \text { Significant at } 0.01 \text { level. } \\
\text { PHQ-9 = Patient Health Questionnaire-9, RSES = Rosenberg Self-Esteem Scale, } \\
\text { SCI = spinal cord injury, STAI = State-Trait Anxiety Inventory, SWLS = Satis- } \\
\text { faction with Life Scale. }\end{array}$} \\
\hline
\end{tabular}

(28.6\%), and controls (38.1\%) were nearly twice as likely to be married as individuals with SCI (17.5\%).

Since the two groups differed based on partnership status, a MANOVA was run with partnership status as the independent variable (single vs partnered) and satisfaction with life, depressive symptoms, self-esteem, and anxiety as the dependent variables. Because this MANOVA was not statistically significant $(F(4,77)=0.48, p=0.75)$, partnership status was not covaried in the analyses comparing the mental health of individuals with SCI with that of controls.

Thus, a MANOVA was conducted in which the independent variable was participant status (patient vs control), and the dependent variables were satisfaction with life, depressive symptoms, self-esteem, and anxiety. The homogeneity of variance assumption was checked using Levene tests, and all were nonsignificant. The overall MANOVA was statistically significant $(F(4,77)=9.66, p<0.001)$, so follow-up ANOVAs were run to determine the precise location of the significant differences between individuals with SCI and able-bodied controls. Individuals with SCI (mean \pm SD: $16.6 \pm 7.0)$ compared with controls $(22.1 \pm$ 6.2) had significantly lower scores for satisfaction with life $(F(1,80)=14.19, p<0.001)$. Additionally, individuals with SCI $(6.3 \pm 4.2)$ compared with able-bodied controls $(4.0 \pm 3.7)$ had significantly higher scores for depressive symptoms $(F(1,80)=7.08, p=0.01)$. However, individuals with SCI (33.9 \pm 4.3$)$ compared with able-bodied controls $(32.2 \pm 4.7)$ did not have significantly different selfesteem scores $(F(1,80)=2.63, p=0.11)$. Individuals with SCI $(34.9 \pm 19.1)$ compared with able-bodied controls $(34.8 \pm 18.4)$ also did not have significantly different anxiety scores $(F(1,80)=0.268, p=0.98)$. See Table 3 for effect sizes.

\section{DISCUSSION}

The purpose of this study was to compare the mental health of individuals with SCI in Colombia with a ablebodied comparison group. As compared with controls, individuals with SCI had lower satisfaction with life and higher depressive symptoms but comparable levels of selfesteem and anxiety. Although there has been extensive research assessing depression in individuals with SCI, few studies have examined satisfaction with life, self-esteem, and anxiety. The vast majority of this research has been conducted in the United States, Western Europe, and other developed countries, with very little consideration for other global regions, like Latin America. As such, a lack of available information exists on the mental health issues faced by individuals with SCI in Colombia or other Latin American countries. The current study is the first to compare the mental health issues faced by persons with SCI with controls in Latin America.

Table 3.

Mental health scores for individuals with spinal cord injury (SCI) and able-bodied controls.

\begin{tabular}{lccccc}
\hline \multicolumn{1}{c}{ Variable } & $\begin{array}{c}\text { Individuals with SCI } \\
(\mathbf{m e a n} \pm \mathbf{S D})\end{array}$ & $\begin{array}{c}\text { Able-Bodied Controls } \\
(\text { mean } \pm \text { SD) }\end{array}$ & F-Statistic & $\boldsymbol{p}$-Value & Cohen $\boldsymbol{d}$ \\
\hline Satisfaction with Life & $16.6 \pm 7.0$ & $22.1 \pm 6.2$ & 14.19 & $<0.01$ & 0.83 \\
Depressive Symptoms & $6.3 \pm 4.2$ & $4.0 \pm 3.7$ & 7.08 & 0.01 & 0.59 \\
Self-Esteem & $33.9 \pm 4.3$ & $32.2 \pm 4.7$ & 2.63 & 0.11 & 0.36 \\
Anxiety & $34.9 \pm 19.1$ & $34.8 \pm 18.4$ & 0.00 & 0.98 & 0.01 \\
\hline
\end{tabular}

Note: Cohen $d$ effect size: $0.20=$ small, $0.50=$ medium, and $0.80=$ large; $p$-values are two-tailed. 
This study's finding that individuals with SCI had significantly lower scores on satisfaction with life suggests that individuals with SCI in Colombia were less satisfied with their lives than able-bodied controls. This finding is consistent with previous research documenting that individuals with SCI experience lower levels of life satisfaction than the general population in other global regions [12]. Similarly, the finding that individuals with SCI had significantly higher scores on the PHQ-9 suggests that depressive symptoms often co-occurred with SCI in the current sample. This finding replicates the results found by Craig et al. that individuals with SCI in Australia experienced more depressive symptoms than age- and sex-matched controls [21]. The current study's findings also conform to the general literature identifying a high prevalence of depressive symptoms among individuals with SCI $[9,12,20,22]$.

An interesting finding, contrary to what was expected in the current study, was that no significant differences emerged between self-esteem and anxiety scores between individuals with SCI and able-bodied controls. While these findings are consistent with previous research documenting no differences in self-esteem between individuals with SCI and controls in the United States [41], other studies have found that individuals with SCI experience poorer self-esteem and higher levels of anxiety than the general population [21]. Despite these mixed results regarding anxiety in individuals with SCI, some researchers have argued that in actuality, only a minority of individuals develop clinically significant anxiety after an SCI [14]. This assertion indeed found support in the current study.

These inconsistencies in the literature could be caused by a number of associated factors. Studies examining individuals with high levels of anxiety after SCI have found that increased anxiety was significantly associated with the traumatic event leading to the injury $[9,21,42]$. This finding bears on the current study in that only 35 percent of the injuries were a result of violence. Although anxiety disorders can also develop as a result of nonviolent trauma, violent events may produce a different constellation of anxiety symptoms than do other more common causes of SCI in Latin America, such as falls [3].

Another associated factor that could account for the lower anxiety scores found may be attributed to the time since injury. In the current study, the average time since injury was $142.6 \mathrm{mo}$, or roughly $12 \mathrm{yr}$. Research has found that although anxiety levels are high immediately following injury during hospitalization, these levels tend to fall below clinical levels after discharge [9]. As such, the presence of lower anxiety scores in individuals with SCI in the current study may be because of the extended time since injury.

While there has been previous research showing that individuals with SCI have lower scores on measures assessing self-esteem [43-47], the current study is consistent with previous research suggesting that the longer the time period since the SCI, the higher the scores measuring self-esteem [43,46-49]. Again, longer time since injury could allow individuals with SCI more time to integrate SCI into their identity, contributing to higher self-esteem scores.

\section{LIMITATIONS AND FUTURE DIRECTIONS}

One limitation to the current study was the limited sample of individuals with SCI and matched controls from Colombia. This sample size may have contributed to a lack of statistical significance for differences in the mental health variables of self-esteem and anxiety, because a power analysis suggested that the current sample would not be able to detect small effects or some medium-sized effects. Limited sample size is especially influential on tests of mean differences when scale scores have high variability. For example, participants had an STAI average score of 34.8 with an SD of 18.6, indicating high variability and obscuring possible mean differences.

A second limitation is characteristic of research on self-esteem, anxiety, and depressive symptoms after SCI more generally. The current study was unable to assess participants' premorbid psychological state and risk prior to SCI. It is possible that participants had specific prior risk factors or protective factors that could influence the onset of mental health problems after injury. Future research should investigate whether other demographic or preinjury variables are associated with the mental health of individuals with SCI.

A third limitation is that more sensitive measures could have been used to assess mental health in this sample. Although the PHQ-9 is established to be a reliable and valid self-report measure of depressive symptoms, it is not as strong an index of depression as a cliniciangiven formal diagnosis would be [50]. Similarly, a measure assessing generalized anxiety disorder or posttraumatic stress disorder could have been used, but since the STAI has been translated into and validated in Spanish, 
the STAI was used for this study. Researchers have argued that specific instruments measuring mental health need to be created for individuals with SCI to take into account physical impairment [14], which would make a valuable addition in future studies.

A fourth limitation is that participants in this study were from Colombia, and thus an appropriate degree of caution should be exercised when generalizing results to all individuals with SCI in Latin America. Additional studies should be conducted in regions outside of the United States, Western Europe, and other developed countries to help broaden the understanding of how SCI affects patients' mental health in diverse global regions.

\section{CONCLUSIONS}

Despite these limitations, the current study highlights some of the mental health issues faced by individuals with SCI in Colombia. In Latin America, SCI rehabilitation services are extremely sparse and rarely include interventions that target mental health postinjury. The current study suggests that satisfaction with life and depressive symptoms are two important variables that cooccur with SCI and warrant attention in rehabilitation services, especially in this region.

\section{ACKNOWLEDGMENTS}

\section{Author Contributions:}

Study concept and design: J. C. Arango-Lasprilla, L. A. Harper, J. A. Coleman, P. B. Perrin, S. L. Olivera, J. L. Perdomo, J. A. Arango. Acquisition of data: S. L. Olivera, J. L. Perdomo, J. A. Arango. Analysis and interpretation of data: L. A. Harper, J. A. Coleman, P. B. Perrin.

Drafting of manuscript: L. A. Harper, J. A. Coleman, P. B. Perrin, J. C. Arango-Lasprilla.

Critical revision of manuscript for important intellectual content:

L. A. Harper, J. A. Coleman, P. B. Perrin.

Statistical analysis: L. A. Harper, J. A. Coleman, P. B. Perrin.

Administrative, technical, or material support: S. L. Olivera,

J. L. Perdomo, J. A. Arango.

Study supervision: J. C. Arango-Lasprilla.

Financial Disclosures: The authors have declared that no competing interests exist.

Funding/Support: This material was unfunded at the time of manuscript preparation.

Institutional Review: This study was approved by the Surcolombia University IRB. Informed consent was obtained from all participants prior to study participation.
Participant Follow-Up: The authors plan to inform participants of the publication of this study.

\section{REFERENCES}

1. Elliott TR, Rivera P. Spinal cord injury. In: Nezu AM, Nezu CM, Geller PA, Weiner IB, editors. Handbook of psychology. Volume 9. Health psychology. New York (NY): Wiley; 2003. p. 415-35.

2. Wyndaele M, Wyndaele JJ. Incidence, prevalence and epidemiology of spinal cord injury: What learns a worldwide literature survey? Spinal Cord. 2006;44(9):523-29.

[PMID: 16389270] http://dx.doi.org/10.1038/sj.sc.3101893

3. Ackery A, Tator C, Krassioukov A. A global perspective on spinal cord injury epidemiology. J Neurotrauma. 2004; 21(10):1355-70. [PMID:15672627] http://dx.doi.org/10.1089/neu.2004.21.1355

4. Fisher TL, Laud PW, Byfield MG, Brown TT, Hayat MJ, Fiedler IG. Sexual health after spinal cord injury: A longitudinal study. Arch Phys Med Rehabil. 2002;83(8):1043-51. [PMID:12161824] http://dx.doi.org/10.1053/apmr.2002.33654

5. Mehta S, Orenczuk S, Hansen KT, Aubut JA, Hitzig SL, Legassic M, Teasell RW; Spinal Cord Injury Rehabilitation Evidence Research Team. An evidence-based review of the effectiveness of cognitive behavioral therapy for psychosocial issues post-spinal cord injury. Rehabil Psychol. 2011; 56(1):15-25. [PMID:21401282] http://dx.doi.org/10.1037/a0022743

6. Vissers M, van den Berg-Emons R, Sluis T, Bergen M, Stam H, Bussmann H. Barriers to and facilitators of everyday physical activity in persons with a spinal cord injury after discharge from the rehabilitation centre. J Rehabil Med. 2008;40(6):461-67. [PMID:18509562]

7. Krause JS. Years to employment after spinal cord injury. Arch Phys Med Rehabil. 2003;84(9):1282-89. [PMID:13680562] http://dx.doi.org/10.1016/S0003-9993(03)00265-X

8. Phelps J, Albo M, Dunn K, Joseph A. Spinal cord injury and sexuality in married or partnered men: activities, function, needs, and predictors of sexual adjustment. Arch Sex Behav. 2001;30(6):591-602. [PMID:11725457] http://dx.doi.org/10.1023/A:1011910900508

9. Kennedy P, Rogers BA. Anxiety and depression after spinal cord injury: A longitudinal analysis. Arch Phys Med Rehabil. 2000;81(7):932-37. [PMID:10896007] http://dx.doi.org/10.1053/apmr.2000.5580

10. Charlifue SW, Gerhart KA. Behavioral and demographic predictors of suicide after traumatic spinal cord injury. 
Arch Phys Med Rehabil. 1991;72(7):488-92. [PMID:2059121]

11. Budh CN, Osteråker AL. Life satisfaction in individuals with a spinal cord injury and pain. Clin Rehabil. 2007; 21(1):89-96. [PMID:17213246] http://dx.doi.org/10.1177/0269215506070313

12. Post MW, van Leeuwen CM. Psychosocial issues in spinal cord injury: A review. Spinal Cord. 2012;50(5):382-89. [PMID:22270190] http://dx.doi.org/10.1038/sc.2011.182

13. Saunders LL, Krause JS, Focht KL. A longitudinal study of depression in survivors of spinal cord injury. Spinal Cord. 2012;50(1):72-77. [PMID:21808257] http://dx.doi.org/10.1038/sc.2011.83

14. Craig A, Tran Y, Middleton J. Psychological morbidity and spinal cord injury: A systematic review. Spinal Cord. 2009; 47(2):108-14. [PMID:18779835] http://dx.doi.org/10.1038/sc.2008.115

15. Dijkers MP, Zanca JM. Factors complicating treatment sessions in spinal cord injury rehabilitation: nature, frequency, and consequences. Arch Phys Med Rehabil. 2013;94(4 Suppl):S115-24. [PMID:23465468] http://dx.doi.org/10.1016/j.apmr.2012.11.047

16. Craig A, Tran Y, Wijesuriya N, Middleton J. Fatigue and tiredness in people with spinal cord injury. J Psychosom Res. 2012;73(3):205-10. [PMID:22850261] http://dx.doi.org/10.1016/j.jpsychores.2012.07.005

17. Lidal IB, Jensen AE, Larsen TW, Stanghelle JK. Fatigue in persons who have lived with spinal cord injury for $>20$ years. Spinal Cord. 2013;51(2):103-8. [PMID:23069767] http://dx.doi.org/10.1038/sc.2012.110

18. van Leeuwen CM, Kraaijeveld S, Lindeman E, Post MW. Associations between psychological factors and quality of life ratings in persons with spinal cord injury: A systematic review. Spinal Cord. 2012;50(3):174-87. [PMID:22042298] http://dx.doi.org/10.1038/sc.2011.120

19. Tate DG, Forchheimer MB, Krause JS, Meade MA, Bombardier $\mathrm{CH}$. Patterns of alcohol and substance use and abuse in persons with spinal cord injury: Risk factors and correlates. Arch Phys Med Rehabil. 2004;85(11):1837-47. [PMID:15520979] http://dx.doi.org/10.1016/j.apmr.2004.02.022

20. Peter C, Müller R, Cieza A, Geyh S. Psychological resources in spinal cord injury: A systematic literature review. Spinal Cord. 2012;50(3):188-201. [PMID:22124343]

http://dx.doi.org/10.1038/sc.2011.125

21. Craig AR, Hancock KM, Dickson HG. A longitudinal investigation into anxiety and depression in the first 2 years following a spinal cord injury. Paraplegia. 1994;32(10): 675-79. [PMID:7831074] http://dx.doi.org/10.1038/sc.1994.109
22. Dryden DM, Saunders LD, Rowe BH, May LA, Yiannakoulias N, Svenson LW, Schopflocher DP, Voaklander DC. Depression following traumatic spinal cord injury. Neuroepidemiology. 2005;25(2):55-61. [PMID:15947491] http://dx.doi.org/10.1159/000086284

23. Kennedy P, Rogers B. Reported quality of life of people with spinal cord injuries: A longitudinal analysis of the first 6 months post-discharge. Spinal Cord. 2000;38(8):498-503. [PMID:10962611] http://dx.doi.org/10.1038/sj.sc.3101021

24. Barbin JM, Ninot G. Outcomes of a skiing program on level and stability of self-esteem and physical self in adults with spinal cord injury. Int J Rehabil Res. 2008;31(1):59-64. [PMID:18277205] http://dx.doi.org/10.1097/MRR.0b013e3282f28e8a

25. Lee BB, Cripps RA, Fitzharris M, Wing PC. The global map for traumatic spinal cord injury epidemiology: Update 2011, global incidence rate. Spinal Cord. 2014;52(2): 110-16. [PMID:23439068] http://dx.doi.org/10.1038/sc.2012.158

26. Gary KW, Nicholls E, Shamburger A, Stevens LF, ArangoLasprilla JC. Do racial and ethnic minority patients fare worse after SCI?: A critical review of the literature. NeuroRehabilitation. 2011;29(3):275-93. [PMID:22142762]

27. Krause JS, Broderick L. Outcomes after spinal cord injury: Comparisons as a function of gender and race and ethnicity. Arch Phys Med Rehabil. 2004;85(3):355-62. [PMID:15031817] http://dx.doi.org/10.1016/S0003-9993(03)00615-4

28. Diener E, Emmons RA, Larsen RJ, Griffin S. The Satisfaction With Life Scale. J Pers Assess. 1985;49(1):71-75. [PMID:16367493] http://dx.doi.org/10.1207/s15327752jpa4901_13

29. Pons D, Atienza FL, Balaguer I, García-Merita ML. Satisfaction with Life Scale: Analysis of factorial invariance for adolescents and elderly persons. Percept Mot Skills. 2000; 91(1):62-68. [PMID:11011872] http://dx.doi.org/10.2466/pms.2000.91.1.62

30. Atienza FL, Pons D, Balaguer I, García-Merita ML. Propiedades psicométricas de la escala de satisfacción con la vida en adolescentes. Psicothema. 2000;12(2):314-20. Spanish.

31. Kroenke K, Spitzer RL, Williams JB. The PHQ-9: Validity of a brief depression severity measure. J Gen Intern Med. 2001;16(9):606-13. [PMID:11556941] http://dx.doi.org/10.1046/j.1525-1497.2001.016009606.x

32. Diez-Quevedo C, Rangil T, Sanchez-Planell L, Kroenke K, Spitzer RL. Validation and utility of the patient health questionnaire in diagnosing mental disorders in 1003 general hospital Spanish inpatients. Psychosom Med. 2001; 63(4):679-86. [PMID:11485122] 
33. Rosenberg M. Conceiving the self. New York (NY): Basic Books; 1979.

34. Schmitt DP, Allik J. Simultaneous administration of the Rosenberg Self-Esteem Scale in 53 nations: Exploring the universal and culture-specific features of global selfesteem. J Pers Soc Psychol. 2005;89(4):623-42.

[PMID:16287423]

http://dx.doi.org/10.1037/0022-3514.89.4.623

35. Anson K, Ponsford J. Coping and emotional adjustment following traumatic brain injury. J Head Trauma Rehabil. 2006;21(3):248-59. [PMID:16717502] http://dx.doi.org/10.1097/00001199-200605000-00005

36. Gray-Little B, Williams VS, Hancock T. An IRT analysis of the Rosenberg Self-Esteem Scale. Pers Soc Psychol Bull. 1997;25(5):445-51.

37. Spielberger CD, Gorsuch RL, Lushene PR, Vagg PR, Jacobs AG. Manual for the State-Trait Anxiety Inventory (form Y) ("self-evaluation questionnaire"). Palo Alto (CA): Consulting Psychologists Press; 1983.

38. Novy DM, Nelson DV, Smith KG, Rogers PA, Rowzee RD. Psychometric comparability of the English- and Spanishlanguage versions of the State-Trait Anxiety Inventory. Hisp J Behav Sci. 1995;17(2):209-24. http://dx.doi.org/10.1177/07399863950172005

39. Spielberger CD, Gonzalez-Reigosa F, Natalicio FS, Natalicio DS. Development of the Spanish edition of the State-Trait Anxiety Inventory. Interamerican J Psychol. 1971;5(3-4): 145-58.

40. American Psychiatric Association; Task Force on DSM-IV. Diagnostic and statistical manual of mental disorders: DSM-IV-TR. 4th ed. Washington (DC): American Psychiatric Association; 2000.

41. Coyle CP, Lesnik-Emas S, Kinney WB. Predicting life satisfaction among adults with spinal cord injuries. Rehabil Psychol. 1994;39(2):95-112.

http://dx.doi.org/10.1037/h0080309

42. Chung MC, Preveza E, Papandreou K, Prevezas N. The relationship between posttraumatic stress disorder following spinal cord injury and locus of control. J Affect Disord. 2006;93(1-3):229-32. [PMID:16647760] http://dx.doi.org/10.1016/j.jad.2006.02.021

43. Nelson A. Normalization: The key to integrating the spinal cord injured into the community. SCI Nurs. 1987;4:3-6.
44. Piazza D, Holcombe J, Foote A, Paul P, Love S, Daffin P. Hope, social support and self-esteem of patients with spinal cord injuries. J Neurosci Nurs. 1991;23(4):224-30.

[PMID:1833481]

http://dx.doi.org/10.1097/01376517-199108000-00008

45. Roy C. Introduction to nursing: An adaptation model. Englewood Cliffs (NJ): Prentice-Hall; 1976.

46. Trieschmann RB. Spinal cord injuries: Psychological, social and vocational rehabilitation. 2nd ed. New York (NY): Demos; 1988.

47. Marini I, Rogers L, Slate JR, Vines C. Self-esteem differences among persons with spinal cord injury. Rehabil Couns Bull. 1995;38(3):198-206.

48. Cook D. Psychological adjustment to spinal cord injury: Incidence of denial, depression, and anxiety. Rehabil Psychol. 1979;26(3):97-104. http://dx.doi.org/10.1037/h0090926

49. Livneh $\mathrm{H}$. A unified approach to existing models of adaptation to disability: A model of adaptation. In: Marinelli RP, Dell Orto AE, editors. The psychological and social impact of disability. 3rd ed. New York (NY): Springer; 1991. p. 181-96.

50. Sakakibara BM, Miller WC, Orenczuk SG, Wolfe DL; SCIRE Research Team. A systematic review of depression and anxiety measures used with individuals with spinal cord injury. Spinal Cord. 2009;47(12):841-51.

[PMID:19621021]

http://dx.doi.org/10.1038/sc.2009.93

Submitted for publication April 8, 2013. Accepted in revised form July 9, 2013.

This article and any supplementary material should be cited as follows:

Harper LA, Coleman JA, Perrin PB, Olivera SL, Perdomo JL, Arango JA, Arango-Lasprilla JC. Comparison of mental health between individuals with spinal cord injury and able-bodied controls in Neiva, Colombia. J Rehabil Res Dev. 2014;51(1):127-36.

http://dx.doi.org/10.1682/JRRD.2013.04.0086

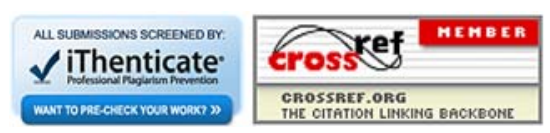


